\title{
NRP MODE TYPING FOR 53 PERSEI: RESULTS FROM
}

\section{VOYAGER PHOTOMETRY}

\author{
MYRON A. SMITH \\ IUE/CSC Observatory, 10000A Aerospace Rd., Lanham-Seabrook MD 20706, USA \\ and \\ LIN HUANG \\ Beijing Observatory, Beijing, China
}

\section{Introduction}

Huang et al. (1994) have conducted an extensive photometric campaign on the prototypical nonradially pulsating (NRP) star 53 Per and confirmed the pair of frequencies at $0.46 \mathrm{cy} \mathrm{d}^{-1}$ (dominant) and $0.60 \mathrm{cy} \mathrm{d} \mathrm{d}^{-1}$ originally reported by BS79. In an analysis of these two modes, SP79 underestimated the effects of the Balmer jump on this star's color variation, leading them to an erroneous conclusion that geometric effects dominate the color variations and also that the modes are described by indices $l=3,-m=3$ and 2. Herein we describe results from Voyager 2 observations obtained during Lin's optical campaign. The amplitudes derived for these Far-UV data using the optical ephemeris provide for the first time a large enough wavelength baseline to discriminate in favor of thermal effects over geometrical ones in producing NRP light variations. In addition, they allow the dominant mode to be constrained to $l=2$ (or 1 ).

\section{Observations}

Eleven $\sim 1 \mathrm{hr}$ observations made over three days in 1991 January were of sufficient quality to permit reliable extraction, binning, calibration, and descattering of UVS Voyager spectra by the techniques of Holberg and Watkins (1992). As expected for bright stars, the deviations of our data from a twosinusoid fit suggest an error of $\pm 3 \%$. We have extracted $100 \AA$ bandpasses centered at $\lambda 1180$ and $\lambda 1585$. The fit to our data gives amplitudes of 0.147 , 0.035 mags. at $\lambda 1180$ and $0.098,0.022$ mags. for $\lambda 1585$, respectively, for the two modes. To determine a modal $l$ identification for 53 Per's primary mode, we can simply ratio the Far-UV amplitudes with Huang et al.'s U, V observations.

\section{Theoretical NRP Light Variations and Conclusions}

In linear adiabatic theory the amplitude of an NRP wave on a star is described by the spherical harmonic function, $\mathrm{Y}_{m}^{l}$. Light variations for all 
Table 1: Light Variation Terms, Theory and Observation

\begin{tabular}{lccccccc}
\hline & $l$ Mode & $\alpha+\beta$ & $\gamma$ & $\gamma F_{T}^{\prime} T_{P}^{\prime} P_{R}^{\prime}$ & Sum & Pred. Rat. & Obs. \\
\hline $\mathrm{V}:$ & 1 & 0 & .6771 & 9.17 & 9.17 & & \\
& 2 & -1.155 & .2878 & 12.35 & 11.20 & & \\
& 3 & -.314 & .0326 & 2.84 & 2.53 & & \\
& & & & & & & $3600 \AA / \mathrm{V}$ \\
$\mathrm{U}:$ & 1 & 0 & .6778 & 15.87 & 15.87 & 1.7 & \\
& 2 & -1.163 & .2896 & 21.47 & 20.31 & 1.8 & $1.7 \pm .2$ \\
& 3 & -.337 & .0348 & 5.24 & 4.90 & 1.9 & \\
& & & & & & & $1565 \AA / \mathrm{V}$ \\
$1565:$ & 1 & 0 & .7138 & 25.4 & 25.4 & 2.9 & \\
& 2 & -1.446 & .3592 & 42.15 & 40.70 & 3.6 & $3.0 \pm 1$. \\
& 3 & -.910 & .0942 & 22.43 & 21.52 & 8.5 & \\
$1180:$ & 1 & & & & & & $1180 \AA / \mathrm{V}$ \\
& 2 & -1.660 & .4094 & 50.83 & 49.17 & 4.4 & $4.6 \pm 1$. \\
& 3 & -1.40 & .1437 & 36.20 & 34.80 & 13.8 & \\
\hline
\end{tabular}

$m$-orders of a given $l$ are the same. They are comprised of three terms: two geometrical (surface area, surface normal) and one compressional/thermal. These terms can be written as disk-integrated combinations of the Legendre and limb darkening functions and their derivatives; BS79 (eqns. 37-39) define them as coefficients $\alpha_{\lambda}, \beta_{\lambda}$, and $\gamma_{\lambda}$. These are computed and shown in cols. 3-4 of Table 1. (Limb darkening coefficients used for their computation are taken from J. Lester, who kindly calculated them from an ATLAS9 model for $53 \mathrm{Per}$ ). Column 5 multiplies col. 4 by parameters representing (via the chain rule) the flux variation per radius change for the temperature term. Col. 6 is the sum (3) + (5) (opposing signs), viz. the predicted amplitude in mags. divided by the fractional radius change, $\epsilon$, and 1.086 .

Table 1 shows the clear dominance of the thermal $(\gamma)$ term for light and color variations because of 53 Per's long period and because of the wavelength sensitivity of $\gamma$. A comparison of the observed and predicted amplitude ratios shows agreement only for $l=2$ (or 1 ). Separate line profile arguments (namely the near absence of RV variations at the half-power points, the large line width variations) can be used to narrow the modal identification to $l=2$. This value is also more in keeping with this star's observed large light variations than is $l=3$.

\section{References}

Huang, L. et al. 1994, these proceedings.

Buta, R. J., and Smith, M. A. 1979, ApJ, 232, 213; "BS79."

Holberg, J., and Watkins, R. 1992, Voyager Data Analysis Hdbk., Vers. 1.2, Univ. Ariz., unpub.

Smith, M. A., and Buta, R. J. 1979, ApJ, 232, L193; “SB79.” 